\title{
STOCHASTIC APPORTIONMENT
}

\author{
Geoffrey Grimmett
}

\begin{abstract}
The problem of how to allocate to states the seats in the US House of Representatives is the most studied instance of what is termed the 'apportionment problem'. We propose a new method of apportionment which is stochastic, which meets the quota condition, and which is fair in the sense of expectations. Two sources of systematic unfairness are identified, firstly the lower bound condition (every state shall receive at least one seat), and secondly the lower quota condition (every state shall receive at least the integer part of its quota).
\end{abstract}

\section{The problem of apportionment}

Ten goats are to be assigned between three brothers in numbers proportional to the ages (in years) of the recipients. Given the integral nature of a goat, it is not generally possible to meet exactly the condition of proportionality, and the resulting 'apportionment problem' is a classic of operational research. The associated literature is extensive, and includes on the one hand discussions of criteria to be used in assessing different schemes, and on the other hand accounts of the properties of specific classes of scheme. A point of especial focus has been the apportionment of the seats in the House of Representatives between the states of the USA. There are currently 50 states (excluding the District of Columbia) and 435 seats, which are to be divided between the states according to the US Constitution [Article I, Section 2] of 1787 thus:

"Representatives ... shall be apportioned among the several States ... according to their respective Numbers ..."

No scheme is proposed in the Constitution, and the Article therein permits a spectrum of interpretation of the phrase 'according to their respective numbers'. Politicians, lawyers, mathematicians and others have been involved since in the cyclical debate of how to apportion the seats.

Although there is little in this note which is specific to the US Congress, we shall, for ease of exposition, use the terminology of the last problem. Our targets here are to survey the general area, and to propose a new method of apportionment which, in a certain way to be made more precise, meets all the usual criteria for such schemes. This new method is a lottery scheme whose implementation uses (pseudo-)random numbers. The scheme is fair so long as no minimal number of

Mathematics Subject Classification (2000). 90B99, 91B32, 62 C99.

Key words and phrases. Apportionment problem, stochastic apportionment.

(c) Geoffrey Grimmett, 1 July 2002 
seats is allocated to all states however small. In the Congressional example, the Constitution contains an additional condition that every state shall receive at least one seat. It is this violation of the principle of proportional representation which renders futile all attempts to obtain a truly fair system in which individuals are equally represented.

We shall in Section 3 describe a new method which meets the so-called quota condition (see Section 2) and gives proportional representation. In Section 4 we present an adaptation of this method for use in situations in which there are lower bounds on the allocations sought. We shall see in Section 4 that, in the presence of a lower bound, the quota condition (see Section 2) provides another source of unfairness.

The established theory of deterministic apportionment is summarised in Section 5. Those interested in learning more of the history and practice of apportionment should consult the excellent book [1] of Balinski and Young.

\section{Quota}

We suppose there are $s$ states with respective populations $\pi_{1}, \pi_{2}, \ldots, \pi_{s}$, and that there are $r$ seats in the House of Representatives. The total population size is $\Pi=\pi_{1}+\pi_{2}+\cdots+\pi_{s}$, and thus the exact quota of seats for state $i$ is $q_{i}=r \pi_{i} / \Pi$. The problem is that $q_{i}$ are not generally integers, whereas representatives are (by axiom) indivisible. We call $\pi=\left(\pi_{1}, \pi_{2}, \ldots, \pi_{s}\right)$ and $q=\left(q_{1}, q_{2}, \ldots, q_{s}\right)$ the population vector and quota vector, respectively. We refer to the pair $(\pi, r)$ as a problem.

An allocation is a vector $\alpha=\left(\alpha_{1}, \alpha_{2}, \ldots, \alpha_{s}\right)$ of non-negative integers with sum $r$. An allocation $\alpha$ is said to 'satisfy quota' if $\alpha_{i} \in\left\{\left\lfloor q_{i}\right\rfloor,\left\lceil q_{i}\right\rceil\right\}$ for all $i$. An allocation is said to 'violate quota' if it does not satisfy quota. Here, $\lfloor x\rfloor$ denotes the greatest integer not exceeding $x$, and $\lceil x\rceil$ denotes the least integer not less than $x$. We speak of $\left\lfloor q_{i}\right\rfloor$ (respectively, $\left\lceil q_{i}\right\rceil$ ) as the lower (respectively, upper) quota for state $i$.

It seems generally (if not universally) accepted that the property of satisfying quota is desirable. It is clear that, for all problems of the above type, there exists necessarily at least one allocation which satisfies quota. This can cease to be the case when further conditions are added. In a variety of situations including that of the US Congress, there is a requirement that the $\alpha_{i}$ be not too small. Let $l=$ $\left(l_{1}, l_{2}, \ldots, l_{s}\right)$ be a given vector of non-negative integers. We say that an allocation $\alpha$ 'has lower bound' $l$ if $\alpha_{i} \geq l_{i}$ for all $i$. Of special interest is the case $l=1$, the vector of ones, not least since the relevant article of the US Constitution requires that

$$
\text { "each State shall have at Least one Representative ..." }
$$

Such a requirement is potentially disturbing since there exist problems $(\pi, r)$ for which no allocation exists satisfying quota and with lower bound 1. For a simple (if extreme) example, consider the case when $\pi=(1,1,7)$ and $r=3$.

It is a simple matter to see that there exists an allocation which satisfies quota and with lower bound $l$ if and only if

$$
l_{i} \leq\left\lceil q_{i}\right\rceil \text { for all } i, \text { and } \sum_{i} \max \left\{l_{i},\left\lfloor q_{i}\right\rfloor\right\} \leq r
$$

(c) Geoffrey Grimmett, 1 July 2002 
The satisfying of quota seems to be regarded as paramount amongst properties of allocations. As noted above, we shall see in Section 4 that, in the presence of a lower bound, the requirement of quota can be a further source of unfairness. There are certain desirable properties of schemes for finding allocations, most prominently that the scheme avoids each of the three 'paradoxes' - Alabama, population, and new-state - to which we shall return in Section 5 .

A word for the novice - in common with other similar problems, one may be tempted to identify many desirable properties of schemes, only to find that no scheme has them all. A well known example of this phenomenon is 'Arrow's Impossibility Theorem', which states that no preference ranking exists for a society which embraces a certain collection of five reasonable axioms (see [4, Chapter 14]).

\section{Stochastic apportionment without lower bounds}

Although lottery schemes have been mentioned briefly in the literature (see, for example, [1]), the established theory is concentrated on deterministic schemes. Our purpose here is to propose a family of stochastic schemes, one in particular, which satisfy quota and which have the advantage of being truly fair and proportional in that each state receives a (possibly random) number of seats having mean value equal to the quota of the state. We will see in Section 4 that this cannot generally be achieved in the presence of lower bounds on allocations, and therefore we make the facilitating assumption for the duration of this section that no lower bound is required.

A random allocation is a vector $A=\left(A_{1}, A_{2}, \ldots, A_{s}\right)$ of non-negative-integervalued random variables with sum $r$. A randomized scheme is a mapping which, to each problem $(\pi, r)$, allocates a probability distribution on the space of appropriate allocations. Otherwise expressed, a randomized scheme results in a random allocation (we shall not spend any time on the choice of probability space, and such like).

There is a subtlety to the notion of a randomized scheme which we discuss briefly. We may seek to apply such a scheme to two given problems, perhaps by applying it twice to the same problem $(\pi, r)$, or perhaps by applying it to $(\pi, r)$ and to another problem $\left(\pi^{\prime}, r^{\prime}\right)$ obtained from $(\pi, r)$ by changing some of the parameters. In so doing, we encounter the question of 'coupling'. That is, since randomized schemes make use of pseudo-random numbers, we shall need to specify whether, at the one extreme, we re-use for the second problem the pseudo-random numbers used already for the first, or, at the other extreme, we make use of 'new' pseudo-random numbers. We discuss this no further at this stage (see, however, the two final paragraphs of this section), since the discussion will concentrate for the moment on the use of randomized schemes for a single problem only.

We say that a random allocation $A$ 'satisfies quota almost surely' if

$$
\mathbb{P}(A \text { satisfies quota })=1 \text {. }
$$

Here, $\mathbb{P}$ denotes probability, and (later) $\mathbb{E}$ denotes expectation. A randomized scheme is said to satisfy quota if the ensuing random allocation satisfies quota almost surely. 
In advance of making a concrete proposal for a randomized scheme, we point out that there exist in general a multiplicity of such schemes which satisfy quota. Let $q_{i}^{\prime}=q_{i}-\left\lfloor q_{i}\right\rfloor$, let $s^{\prime}=\left|\left\{i: q_{i}^{\prime}>0\right\}\right|$, and let $r^{\prime}=r-\sum_{i}\left\lfloor q_{i}\right\rfloor$. We call $q^{\prime}=\left(q_{i}^{\prime}: 1 \leq i \leq s\right)$ the fractional-quota vector. Since state $i$ receives $\left\lfloor q_{i}\right\rfloor$ seats of right, we are required only to allocate the remaining $r^{\prime}$ seats amongst the $s^{\prime}$ 'unsatisfied' states. This may be done in $\left(\begin{array}{c}s^{\prime} \\ r^{\prime}\end{array}\right)$ ways, to each of which we must assign a probability. If we require in addition that the scheme be fair in the sense that the ensuing allocation $A$ satisfies $\mathbb{E}\left(A_{i}\right)=q_{i}$ for all $i$, then we obtain thus a set of $s^{\prime}$ constraints. Thus the space of such randomized schemes may have up to $\left(\begin{array}{c}s^{\prime} \\ r^{\prime}\end{array}\right)-s^{\prime}$ degrees of freedom. In the special cases $r^{\prime}=1, r^{\prime}=s^{\prime}-1$, there is a unique randomized scheme which is fair and satisfies quota.

We turn now to our concrete proposal. There are three steps.

I. We permute at random the labels of the states.

This is proposed since the scheme which follows depends on the labelling of the states (that is, on the indices of the $\pi_{i}$ ), and it seems desirable to reduce to a minimum any correlations which depend on this extraneous element. In the Congressional example, it would arguably not be right for the allocation to Alabama to depend systematically to a greater degree on that to Alaska than that to Maine. For ease of presentation, we shall in the following not change the notation $\pi_{i}$, but shall assume that $\pi$ is the population vector after permuting at random.

II. We provisionally allocate $\left\lfloor q_{i}\right\rfloor$ seats to state $i$.

This is prompted by the minimal quota for each state, and leaves so far unallocated a certain number $r^{\prime}=r-\sum_{i}\left\lfloor q_{i}\right\rfloor$ of seats. Let $q_{i}^{\prime}=q_{i}-\left\lfloor q_{i}\right\rfloor$, as before.

III. Let $U$ be a random variable with the uniform distribution on $[0,1]$, and let $Q_{i}=U+\sum_{j=1}^{i} q_{i}^{\prime}$. Let $A_{i}^{\prime}$ be the indicator function of the event that the interval $\left[Q_{i-1}, Q_{i}\right)$ contains an integer. We allocate a further $A_{i}^{\prime}$ seats to state $i$.

State $i$ receives a total of $\left\lfloor q_{i}\right\rfloor+A_{i}^{\prime}=A_{i}$ seats. The total number of seats allocated at step III equals the length of the interval $\left[Q_{0}, Q_{s}\right)$, which equals $\sum_{i}\left\{q_{i}-\left\lfloor q_{i}\right\rfloor\right\}=r^{\prime}$, whence $A$ is an allocation (that is, it has sum $r$ ). It is evident that $\left\lfloor q_{i}\right\rfloor \leq A_{i} \leq\left\lceil q_{i}\right\rceil$, whence $A$ satisfies quota. In addition, each $Q_{j}$, when reduced modulo 1 , is uniformly distributed on the interval $[0,1]$. Therefore, $\mathbb{E}\left(A_{i}^{\prime}\right)$ equals the length of the interval $\left[Q_{i-1}, Q_{i}\right)$, which is to say that $\mathbb{E}\left(A_{i}^{\prime}\right)=Q_{i}-Q_{i-1}=q_{i}^{\prime}$ and hence $\mathbb{E}\left(A_{i}\right)=q_{i}$. We summarise this by saying that the scheme satisfies quota, and is fair in the sense that the mean number of seats per head of population is constant between states.

We offer no justification for this scheme apart from fairness and ease of implementation. We caution against adopting any randomized scheme without a proof of fairness, and as an example we summarise one arguably reasonable but definitely unfair scheme for allocating the remaining $r^{\prime}$ seats.

Conditional sampling. We select independent indices $I_{t}, 1 \leq t \leq r^{\prime}$, each $I_{t}$ having distribution $\mathbb{P}\left(I_{t}=i\right)=q_{i}^{\prime} / \sum_{i} q_{i}^{\prime}$, and we consider a random vector $J=$ $\left(J_{1}, J_{2}, \ldots, J_{r^{\prime}}\right)$ having the probability distribution of $I_{1}, I_{2}, \ldots, I_{r^{\prime}}$ conditional on $I_{u} \neq I_{v}$ for all $u \neq v$. We allocate one extra seat to those states having indices $J_{1}, J_{2}, \ldots, J_{r^{\prime}}$. It is the case that

$$
\mathbb{E}\left|\left\{t: I_{t}=i\right\}\right|=q_{i}^{\prime} \text { for all } i,
$$

(c) Geoffrey Grimmett, 1 July 2002 
but the same statement is generally false with $I$ replaced by $J$. This is easily seen when $r^{\prime}$ and $s^{\prime}$ are large. The conditioning amounts to a large deviation, and the distribution of $J$ may be close to an appropriately tilted distribution (see $[3$, Section $5.11])$.

We close this section with some remarks on the use of pseudo-random numbers. It will be argued that the fairness of a stochastic scheme may not be evident to the population. It seems that politicians may be less willing than the people to accept such a scheme, since politicians are very sensitive to the marginal value to a party of a single seat. Lotteries are already in wide use in areas having impact on individuals, not least in state-accredited systems for raising money for so-called 'good causes'. As a further example, the allocation of individuals to the control group of a medical trial is usually done by lottery, and such a decision may be a matter of life or death. See [5] for an extended discussion of the drawing of lots.

When applying a randomized scheme to two or more problems, one needs to decide whether or not to re-sample the required pseudo-random numbers. The expectations under study remain the same, but the external perception of fairness is likely to be greater if the roulette wheel is spun afresh. A statistical virtue of re-sampling is the reduction of variances.

\section{Stochastic apportionment with lower bounds}

We consider in this section the problem of finding an allocation which satisfies quota and is subject to a lower bound $l$. Let $l=\left(l_{1}, l_{2}, \ldots, l_{s}\right)$ be a vector of non-negative integers satisfying $l_{i} \leq\left\lceil q_{i}\right\rceil$ for all $i$. We assume in addition that $\sum_{i} l_{i} \leq r$, since otherwise there exists no allocation with lower bound $l$ and sum $r$. We call a state small if $q_{i}<l_{i}$, and we note that the lower-bound condition is tantamount to favouring the small states. It follows that, whenever there exist small states, then the other states are at a disadvantage, and there can exist no scheme which is fair. But how fair a randomized scheme can be achieved?

Let $I_{-}=\left\{i: q_{i}<l_{i}\right\}$ be the set of small states, and also $I_{=}=\left\{i: q_{i}=l_{i}\right\}$ and $I_{+}=\left\{i: q_{i}>l_{i}\right\}$. We assume that there exists at least one small state, in that $I_{-} \neq \varnothing$, since otherwise there is no new problem. Since $\sum_{i} l_{i} \leq r$, it must be the case that $I_{+} \neq \varnothing$. For $i \in I_{-} \cup I_{=}$, we allocate to state $i$ exactly $l_{i}$ seats, and we write $\mu=r-\sum_{i \in I_{-} \cup I_{=}} l_{i}$ for the number of remaining seats. Note that $\sum_{i \in I_{+}} q_{i}>\mu$, whence no fair allocation can exist.

In allocating seats to states in $I_{+}$, we seek a guiding principle, and we propose the principle of equality of representation amongst states in $I_{+}$.

The scheme of Section 3 (particularly step III thereof) is based on the quota vector $q$ and the fractional-quota vector $q^{\prime}$. Since the allocations to states in $I_{-} \cup I_{=}$ have already been determined, we are directed by the above principle to seek a new quota vector $Q=\left(Q_{i}: i \in I_{+}\right)$such that:

(a) $\sum_{i \in I_{+}} Q_{i}=\mu$,

(b) the mean number of representatives per head of population is constant between states in $I_{+}$, which is to say that there exists a constant $\gamma$ such that

$$
\frac{Q_{i}}{q_{i}}=\gamma \quad \text { for all } i \in I_{+},
$$

(c) Geoffrey Grimmett, 1 July 2002 
(c) $\left\lfloor q_{i}\right\rfloor \leq Q_{i} \leq\left\lceil q_{i}\right\rceil$ for all $i \in I_{+}$.

Following consideration of the lower bound condition, we shall require also that

(d) $Q_{i} \geq l_{i}$ for all $i \in I_{+}$.

Note that (c) implies (d) since, by (c),

$$
Q_{i} \geq\left\lfloor q_{i}\right\rfloor \geq l_{i} \quad \text { for } i \in I_{+} .
$$

We next investigate conditions under which (a), (b), (c) may be achieved simultaneously. Assume that (a) and (b) hold. We sum (4.1) over $i \in I_{+}$to obtain by (a) that

$$
\gamma=\frac{\sum_{i \in I_{+}} Q_{i}}{\sum_{i \in I_{+}} q_{i}}=\frac{\mu}{\sum_{i \in I_{+}} q_{i}} .
$$

It follows that (c) is satisfied if and only if

$$
Q_{i}=\gamma q_{i} \geq\left\lfloor q_{i}\right\rfloor \quad \text { for all } i \in I_{+} .
$$

If (c) holds, which is equivalent to $\left\lfloor Q_{i}\right\rfloor=\left\lfloor q_{i}\right\rfloor$ for $i \in I_{+}$, then we proceed by applying the algorithm of the last section thus. For $i \in I_{+}$, we allocate $\left\lfloor q_{i}\right\rfloor$ seats to state $i$. Then we apply step III to the new fractional-quota vector $Q^{\prime}=\left(Q_{i}-\left\lfloor Q_{i}\right\rfloor\right.$ : $i \in I_{+}$) with (by (a)) $\mu-\sum_{i \in I_{+}}\left\lfloor q_{i}\right\rfloor$ seats. The outcome is a random allocation which satisfies quota and which is fair when restricted to the states in $I_{+}$.

Suppose that (c) does not hold, in that there exists $i \in I_{+}$such that $Q_{i}<\left\lfloor q_{i}\right\rfloor$. One strategy would be to apply the scheme of the last section to the quota vector $Q$, obtaining thereby a random allocation $A$ satisfying $\mathbb{E}\left(A_{i}\right)=Q_{i}$ for each $i \in I_{+}$. If this random allocation does not satisfy quota, then the scheme has failed. There is however a certain probability that quota is satisfied. By counting the mean number of states whose allocation does not satisfy the respective quota, we find that

$$
\mathbb{P}(A \text { does not satisfy quota }) \leq \sum_{i \in I_{+}} \max \left\{1,\left\lfloor q_{i}\right\rfloor-Q_{i}\right\} 1_{\left\{Q_{i}<\left\lfloor q_{i}\right\rfloor\right\}},
$$

where $1_{A}$ denotes the indicator function of the event $A$. Equality holds in (4.4) if there exists at most one state $i$ with $Q_{i}<\left\lfloor q_{i}\right\rfloor$.

Let us suppose that (c) does not hold but that the outcome $A$ satisfies quota. A slightly subtle point is that, conditional on this event, $A$ is not fair. This is so because the conditioning changes the expectations. In summary, the scheme work with the quota vector $Q$ repeatedly until one obtains a random allocation which satisfies quota - is not a fair scheme.

A feasible line of enquiry which we have not pursued is to postulate probabilistic models for problems, and to calculate the probability for such a model that the above scheme results in an allocation which does not satisfy quota. In certain circumstances, the 'law of anomalous numbers' (see $[2,3]$ ) could be used as a basis for such a model.

If the satisfying of quota is paramount, then one must accept a further degree of unfairness, and we propose the following. If $Q_{i}<\left\lfloor q_{i}\right\rfloor$, we allocate to state $i$ exactly 
$\left\lfloor q_{i}\right\rfloor$ seats. Writing $J_{+}=\left\{i \in I_{+}: Q_{i} \geq\left\lfloor q_{i}\right\rfloor\right\}$, we now iterate the above process restricted to the states in $J_{+}$. That is, we seek, as above, numbers $R_{i}, i \in J_{+}$, such that:

(a') $\sum_{i \in J_{+}} R_{i}=\mu-\sum_{i \in I_{+} \backslash J_{+}}\left\lfloor q_{i}\right\rfloor$,

$\left(b^{\prime}\right)$ the mean number of representatives per head of population is constant between states in $J_{+}$, in that there exists a constant $\gamma^{\prime}$ such that

$$
\frac{R_{i}}{q_{i}}=\gamma^{\prime} \quad \text { for all } i \in J_{+},
$$

$\left(\mathrm{c}^{\prime}\right)\left\lfloor q_{i}\right\rfloor \leq R_{i} \leq\left\lceil q_{i}\right\rceil$ for all $i \in J_{+}$.

We find this time from $\left(\mathrm{a}^{\prime}\right)$ and $\left(\mathrm{b}^{\prime}\right)$ that $R_{i}=\gamma^{\prime} q_{i}$ where

$$
\gamma^{\prime}=\frac{\mu-\sum_{i \in I_{+} \backslash J_{+}}\left\lfloor q_{i}\right\rfloor}{\sum_{i \in J_{+}} q_{i}} .
$$

If $R_{i} \geq\left\lfloor q_{i}\right\rfloor$ for all $i \in J_{+}$, we apply the scheme to the new quota vector $R=\left(R_{i}\right.$ : $\left.i \in J_{+}\right)$. Otherwise, we note that any state $i$ with $R_{i}<\left\lfloor q_{i}\right\rfloor$ must be handled in a way which will disfavour those remaining, namely by allocating to it $\left\lfloor q_{i}\right\rfloor$ seats.

This scheme, when iterated to reach a conclusion, identifies different levels of unfairness in its consecutive applications of the principle of equality of representation amongst the remaining states. Note that it terminates with a random allocation which satisfies quota if and only if there exists an allocation which satisfies both quota and the lower bound.

We emphasize that, in the presence of a lower bound, the principle of satisfying quota is another potential source of unfairness. The upper quota presents no problem, but the lower quota can indeed be problematic.

Let us apply the above argument in the Congressional example, with $l=1$. In the following table are listed those states $i$ which, in the nine ten-yearly apportionments of 1920-2000, have $Q_{i}<\left\lfloor q_{i}\right\rfloor$, these being the apportionments with the current House size of 435. In just four of these apportionments, namely those of 1920/1950/1970/2000, do there exist states $i$ with $Q_{i}<\left\lfloor q_{i}\right\rfloor$, and in each such case there is only a small probability that the proposed scheme results in an allocation based on $Q$ which does not satisfy quota. For example, in 1950, the ensuing allocation could only fail to satisfy quota if New York were allocated 42 seats rather than 43 , an event of probability $\left\lfloor q_{i}\right\rfloor-Q_{i}=43-42.962=0.038$, where $i$ is the index of New York. The corresponding probability for the year 2000 is $\left\lfloor q_{j}\right\rfloor-Q_{j}=19-18.999=0.001$, where $j$ is the index of Pennsylvania. A similar calculation is valid for the other years, and in each case the resulting probability is small. Based on this empirical evidence, we claim that the proposed scheme, based on $Q$, is likely to result in an apportionment of the House of Representatives which satisfies quota.

(c) Geoffrey Grimmett, 1 July 2002 


\begin{tabular}{ccccc} 
Year & \# small states & states with $Q_{i}<\left\lfloor q_{i}\right\rfloor$ & original quota $q_{i}$ & new quota $Q_{i}$ \\
1920 & 3 & Pennsylvania & 36.053 & 35.973 \\
& \multicolumn{5}{c}{ Florida } & 4.004 & 3.995 \\
1930 & 3 & none & & \\
1940 & 3 & none & & \\
1950 & 3 & New York & 43.038 & 42.962 \\
1960 & 4 & none & & \\
1970 & 3 & Virginia & 10.000 & 9.984 \\
1980 & 3 & none & & \\
1990 & 3 & none & & 18.999 \\
2000 & 4 & Pennsylvania & 19.013 &
\end{tabular}

Table 1. For the apportionments of 1920-2000, this is a list of states $i$ for which $Q_{i}<\left\lfloor q_{i}\right\rfloor$. The entries are derived from data to be found in $[1]$.

However, in these years 1920/1950/1970/2000, such an outome would be slightly unfair to the other states. Suppose, instead, we acknowledge this unfairness explicitly by automatically allocating to any state $i$ with $Q_{i}<\left\lfloor q_{i}\right\rfloor$ the number $\left\lfloor q_{i}\right\rfloor$ seats. In each case, when we calculate the amended quota vector $R=\left(R_{i}: i \in J_{+}\right)$, we find that every $R_{i}$ satisfies $R_{i} \geq\left\lfloor q_{i}\right\rfloor$. The outcome of the composite scheme is therefore an allocation which favours firstly the small states, and secondly any state $i$ with $Q_{i}<\left\lfloor q_{i}\right\rfloor$, and then proceeds to allocate seats to the remaining states in a way which gives equality of representation between them.

Finally we note the existence of an alternative method which might in principle be followed. This is easier to apply but violates the condition of fairness, and is as follows. One reduces the original fractional-quota vector $\left(q_{i}^{\prime}: i \in I_{+}\right)$, by a constant factor, that is, one works with a new fractional-quota vector $\left(q_{i}^{\prime \prime}: i \in I_{+}\right)$given by $q_{i}^{\prime \prime}=\delta q_{i}^{\prime}$ satisfying

$$
\sum_{i \in I_{+}}\left\{\left\lfloor q_{i}\right\rfloor+q_{i}^{\prime \prime}\right\}=\mu
$$

\section{Deterministic apportionment}

It is conventional to apportion the House of Representatives using an algorithm which is deterministic rather than stochastic. The book [1] is an excellent account of theory and practice for mathematicians and non-mathematicians alike, and the reader is referred to it for a full account. No attempt is made here to do more than to summarise the situation.

Let us assume that there is no lower bound. The discussion in the literature has concentrated largely on a category of schemes termed 'divisor methods'. Let $\delta:\{0,1,2, \ldots\} \rightarrow \mathbb{R}$ be a given function satisfying $b \leq \delta(b) \leq b+1$ for all $b$. Let $\lambda$ be a parameter taking positive values, to be thought of as the notional number of head of population to be represented by each representative. The population $\pi_{i}$ of state $i$ is divided by $\lambda$ to obtain the $\lambda$-quota $\pi_{i} / \lambda$ of seats for that state. At the 
first stage, we find the $\lambda$-allocation to state $i$, defined as

$$
\alpha_{i}(\lambda)= \begin{cases}\left\lfloor\pi_{i} / \lambda\right\rfloor & \text { if } \pi_{i} / \lambda \leq \delta\left(\left\lfloor\pi_{i} / \lambda\right\rfloor\right), \\ \left\lfloor\pi_{i} / \lambda\right\rfloor+1 & \text { if } \pi_{i} / \lambda>\delta\left(\left\lfloor\pi_{i} / \lambda\right\rfloor\right) .\end{cases}
$$

When equality holds in that $\pi_{i} / \lambda=\delta\left(\left\lfloor\pi_{i} / \lambda\right\rfloor\right)$, we have set $\alpha_{i}(\lambda)=\left\lfloor\pi_{i} / \lambda\right\rfloor$ for the sake of definitiveness, but it is important only that some definite rule be followed. The resulting $\lambda$-allocation $\alpha(\lambda)=\left(\alpha_{i}(\lambda): 1 \leq i \leq s\right)$ does not generally sum to the house size $r$. At the second stage, we 'tune' $\lambda$ until we find $\bar{\lambda}$ such that $\sum_{i} \alpha_{i}(\bar{\lambda})=r$, and we output the allocation $\alpha(\bar{\lambda})$.

There is an infinity of possible choices for the function $\delta$, of which the following instances have been studied.

\section{Name}

Smallest divisors

Harmonic means

Equal proportions

Major fractions

Greatest divisors

\section{Originator}

Adams (1832)

Dean $(1832)$

Hill (1911)

Webster (1832)

Jefferson (1792)
The function $\delta(b)$

$b$
$2 /\left\{b^{-1}+(b+1)^{-1}\right\}$
$\sqrt{b(b+1)}$
$b+\frac{1}{2}$
$b+1$

Table 2. The five principal divisor methods ordered by increasing $\delta$.

A sixth scheme, termed the method of Hamilton (1792) or the 'method of largest remainders', is as follows. A state with quota $q_{i}$ receives by right $\left\lfloor q_{i}\right\rfloor$ seats. The remaining $r-\sum_{i}\left\lfloor q_{i}\right\rfloor$ seats are allocated to those $r-\sum_{i}\left\lfloor q_{i}\right\rfloor$ states $j$ with largest remainders $q_{j}-\left\lfloor q_{j}\right\rfloor$.

One seeks principles which enable distinctions to be drawn between these six schemes. In common with other instances in operational research, one can be overprincipled. Every method has its drawbacks, and to seek the perfect system can be to eliminate all possibilities. A detailed and informative discussion is to be found in [1], from which a few points are extracted here.

A scheme is called population monotone if: for all $i \neq j$, when individuals move from state $i$ to state $j$, then $i$ should not get more seats and $j$ fewer. We have from [1, Thm 6.1] that no scheme exists which is both population monotone and necessarily satisfies quota. Furthermore, (see [1, Prop. 6.4]), Jefferson's 'greatest divisor' method is the only population monotone method which stays above lower quota (in that the ensuing allocation $\alpha$ necessarily satisfies $\alpha_{i} \geq\left\lfloor q_{i}\right\rfloor$ for all $i$ ), and Adams' 'smallest divisor' method is the only population monotone scheme which stays below upper quota (in that $\alpha_{i} \leq\left\lceil q_{i}\right\rceil$ for all $i$ ).

A point of focus is the degree to which a scheme favours large over small states, There are various ways of measuring such bias, both theoretical and empirical, and the case is made in $[1,6]$ that Webster's 'major fraction' method is the least biased in this regard.

A scheme is said to be 'house monotone' if no state's allocation diminishes when the size $r$ of the house increases. A scheme which is not house monotone is said to suffer from the 'Alabama paradox'. It is considered desirable that a scheme be house monotone. Other desirable features of schemes include the absence of 
what are known as the 'population paradox' (in a growing population, state $i$ can grow faster than state $j$, and yet lose a seat to $j$ ), and the 'new-states paradox' (a new state may join the union, with an appropriate number of new seats, but the allocations between the original states change).

One may ask in what sense does the stochastic apportionment scheme of Section 3 meet these requirements. The latter scheme is population monotone in a stochastic sense, which is to say that, when state $i$ loses people to state $j$, the number of seats allocated to $i$ (respectively, $j$ ) is stochastically non-increasing (respectively, non-decreasing). (See [3, Section 4.12] for a definition of stochastic ordering.) In a similar stochastic sense, the scheme is house monotone.

Finally we consider the case of deterministic schemes in situations where there is a non-trivial lower bound on the allocation sought. As said already, there may exist no allocation which satisfies quota and the lower bound, and even if there exists such an allocation, there will generally exist no scheme which is fair across the board. The method used currently for apportioning the House of Representatives is to allocate one seat to each state however small (in 2000 there were just four states whose quotas were smaller than one) and then to apply the method of equal proportions to those states whose 'residual' quotas are strictly positive. The outcomes of this scheme have, fortunately in recent decades, been allocations which have satisfied quota. See [1, Appendix B] for further information.

Acknowledgements. The author thanks Graeme Milton for telling him of the apportionment problem, and Richard Weber for discussing it with him and for help with related references. Of great value have been certain documents available on the web, including writings of Lawrence R. Ernst, H. Peyton Young, and Roman Shapiro.

\section{REFERENCES}

1. Balinksi, M. L., Young, H. P., Fair Representation, Meeting the Ideal of One Person, One Vote, 2nd edn, The Brookings Institution, 2001.

2. Feller, W., An Introduction to Probability Theory and its Applications, Vol. 2, 2nd edn, Wiley, New York, 1971.

3. Grimmett, G. R., Stirzaker, D. R., Probability and Random Processes, 3rd edn, Oxford University Press, Oxford, 2001.

4. Luce, R. D., Raiffa, H., Games and Decisions, Dover, New York, 1989.

5. Silverman, W. A., Chalmers, I., Casting and drawing lots, Controlled Trials from History (Chalmers, I., Milne, I., Tröhler, U., eds.), www.rcpe.ac.uk/controlled_trials, 2001.

6. Young, H. P., Dividing the house: Why Congress should reinstate an old apportionment formula, Policy Brief \#88 (2001), The Brookings Institution, www.brook.edu/dybdocroot/comm/ policybriefs/pb88.htm.

Statistical Laboratory, University of Cambridge, Wilberforce Road, Cambridge CB3 0WB, United Kingdom

E-mail: g.r.grimmett@statslab.cam.ac.uk

$U R L:$ http://www.statslab.cam.ac.uk/ grg/ 